\title{
Faut-il systématiquement recourir à une "imagerie de luxe" pour l'avulsion chirurgicale des dents de sagesse mandibulaires ? Résultats d'une analyse sur 285 cas
}

\author{
Boukais H, Taright S, Zerrouki W, Belguidoum M \\ Service de Pathologie et Chirurgie buccale, CHU Béni-Méssous, Faculté de Médecine, Alger, \\ Algérie \\ h.boukais@yahoo.fr
}

L'avulsion des dents de sagesse mandibulaires constitue un acte fréquent en chirurgie buccale. Comme avant tout acte chirurgical, le bilan préopératoire doit comporter un examen radiologique destiné à connaitre la position exacte et de la dent de sagesse par rapport aux éléments anatomiques de voisinage (nerf alvéolaire inférieur principalement).

Dans la littérature internationale, la tendance actuelle est la systématisation des incidences radiologiques "de luxe", comme le scanner. Ces auteurs pensent que le recours à ces incidences permet de prévenir la lésion accidentelle du nerf alvéolaire inférieur lors de l'avulsion chirurgicale des dents de sagesse mandibulaires.

L'analyse rétrospective et prospective des dossiers de 285 patients pris en charge pour l'avulsion chirurgicale de dents de sagesse mandibulaires incluses ou enclavées, a permis de bien préciser le recours à ces explorations radiologiques "de luxe", et de limiter les indications aux seuls cas où elle est réellement justifiée. Très souvent, les données radiologiques des clichés rétro-alvéolaires, complétées par les données de l'orthopantomographie, et le recours à une technique chirurgicale appropriée et spécifique, ont permis dans bien des cas d'éviter les lésions nerveuses au cours de l'avulsion de ces dents.

Par ailleurs, le recours systématique à ces incidences, en plus de leur coût élevé, est source d'une irradiation potentielle des structures maxillaires et dentaires, sans pour autant améliorer le rapport bénéfice/risques. 